\title{
José Arnaldo Márquez y la generación romántica
}

\author{
Sonia LuZ CARrillo \\ Universidad Nacional Mayor de San Marcos \\ soniluz@hotmail.com

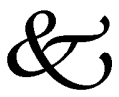

\begin{abstract}
Resumen
En la etapa de la literatura peruana correspondiente al Romanticismo, los nombres de Carlos Augusto Salaverry y Ricardo Palma han concitado, hasta hoy, la mayor atención. El propósito de este artículo es destacar los méritos de un poeta romántico poco estudiado como Arnaldo Márquez quien, dentro de esa vertiente, desarrolló una singular veta humanitarista y socializante. El artículo se completa con una revisión del movimiento romántico en el Perú.
\end{abstract}

Palabras claves: Romanticismo peruano, Arnaldo Márquez, Romanticismo filosófico.

\begin{abstract}
In the stage of the Peruvian literature corresponding to the Romanticisimo, the names of Carlos Augusto Salaverry and Ricardo Palma have attracted, up to this day the greatest interest among scholars on Romanticism. The intention of this article is to emphasize the merits of a little knwon romantic poet Arnaldo Márquez, who developed a singular humanitarian and socializing trait. The article is completed by a review of the romantic movement in Peru.
\end{abstract}

Key words: Peruvian Romanticism, Arnaldo Márquez, Philosophical Romanticism. 


\section{Introducción}

En primer lugar - y al margen de la valoración de los logros artísticos - los estudios literarios coinciden en señalar como característica de los románticos peruanos el hecho de haber constituido un grupo con una clara conciencia: la de integrar una generación literaria y la de haber tenido, además, la noción moderna de arte como fenómeno autónomo, único, con sus exigencias de reflexión, estudio y experimentación, lo que los llevó a interesarse e investigar a los tratadistas de literatura de otras latitudes .

El gobierno del Mariscal Castilla y la Guerra contra España como experiencia común, una actitud de grupo ante los sucesos, similar formación literaria, cercanía en las fechas de sus nacimientos, existencia de líderes y de un lenguaje generacional, aparecen como los rasgos del llamado grupo de los románticos. ${ }^{1}$ A esto se une su "búsqueda de modernidad... (y) la urgencia de internacionalizar la literatura peruana" (Cornejo Polar, 1989: 50), sin dejar por ello de "nacionalizar" la herencia del pasado colonial (Cornejo Polar 1989:89). Con estos rasgos, aparecen vida y obras de estos escritores peruanos de mediados de siglo XIX, en el marco del Romanticismo hispanoamericano.

\section{El romanticismo en hispanoamérica}

El movimiento que se gestara a finales del siglo XVIII en Europa, se expande hacia toda Hispanoamérica en la primera mitad del siglo XIX y se caracteriza por la preeminencia del yo y el mundo subjetivo del autor frente a la realidad objetiva; enarbola el ideal de la libertad individual y muestra una fuerte tendencia al pesimismo así como a la exaltación del sentimiento frente al yugo de la "diosa razón”, y, fiel a su raíz liberal - fruto de la Revolución Francesa - promueve los ideales democráticos y su interés por los rasgos singulares deviene en búsqueda de lo nacional o particular de los pueblos al mismo tiempo que confía

1 La enumeración de estos requisitos para el reconocimiento de una Generación, tales como coincidencia de nacimiento, acontecimiento o experiencia generacional, relaciones interpersonales, existencia de un maestro o líder, homogeneidad de influencias literarias y existencia de un lenguaje generacional, han sido elaborados desde Dilthey, Pinder, hasta Julius Peterson (1930) a quien cita Francisco Mota en su trabajo Poetas españoles de la generación del 27, La Habana : Biblioteca Básica de Literatura española, 1977. 
en el progreso del hombre y la ciencia. Para Anderson (1995: 237) "el romanticismo criollo fue más una actividad civilizadora que escuela de bellas letras ".

En el campo literario - recordemos - sus primeras manifestaciones se dan alrededor de 1770 , en Alemania, cuando un grupo de escritores, que se denomina a sí mismo como Sturn un drang (Tempestad e ímpetu), emerge con la voluntad de renovar la vida cultural y artística y liberarla de los cánones impuestos por el Neoclasicismo. Voluntad que se expande de Alemania a otras naciones como Francia, desde donde llegará a España y de allí se proyectará a nuestros países.

En el ámbito de América hispana "el romanticismo se nutrió en unos casos del ejemplo francés, en otros de la literatura inglesa y en otros siguió el molde español” (Escobar, 1958:34). Argentina es el territorio en el que se consolida más precozmente el romanticismo. Escobar refiere la polémica que en 1842 sostuvieran Andrés Bello, poeta y filólogo venezolano, y Domingo Faustino Sarmiento, educador y político argentino, "aquella polémica reviste trascendencia en la historia literaria de Hispanoamérica... en ella se encontraron Bello, traductor de Hugo y Byron, además de redactor de la más autorizada Gramática de la Lengua Castellana, que significativamente dedicó a los españoles de América, y de otra parte Sarmiento, paradigma del romanticismo argentino, capitán del grupo conocido con el nombre de los proscritos, al que tocó desarrollar parte de la más valiosa producción en el romanticismo de nuestro continente" (Escobar, 1958:35-36).

Desde la Emancipación, diversos autores procedentes del Neoclasicismo se inscriben dentro del romanticismo"ya por su apasionamiento liberal o patriótico, ya por la carga de sensibilidad individualista, ya por el color regional que va filtrándose entre las frías formas y las tendencias rectoras del Clasicismo dieciochesco”. Entre estos autores Tamayo menciona a Bello, Heredia y resalta la presencia de Mariano Melgar (1992 II: 466).

\section{El romanticismo en el Perú}

No obstante estas presencias precursoras, especialmente la de Melgar, el Romanticismo peruano fue un fenómeno tardío. Y respecto a 
la calidad de sus productos, existe coincidencia en las apreciaciones: Más allá de su contenido ético, al Romanticismo peruano - con las excepciones del caso - le faltó rigor. Así, Basadre señala que :"la corriente romántica creciente entre 1851 y 1854, presentó en el Perú características similares a las que tuvo en otros países americanos y también de Europa meridional” y añade que:"aunque escribieran superficial o retóricamente, los románticos peruanos expresaron en conjunto, cada uno a su manera y dentro de las limitaciones de sus obras...el culto al amor idealista y al dolor ; la angustia ante la vida, la muerte, dios, el destino y el alma ; la atracción hacia lugares exóticos, la preocupación nacional y por el pueblo ; la fe en la libertad, la igualdad, la dignidad humana ; el anhelo de una realidad superior al mundo circundante que negaba las más nobles aspiraciones del espíritu humano”. De otro lado, menciona como nota distintiva: "estos literatos escribieron, criticaron y debatieron juntos” (Basadre 1965: IV 65).

Ricardo Palma ayuda a comprender la atmósfera cultural y literaria del momento romántico al relatar en La bohemia de mi tiempo lo siguiente: "De 1848 a 1860 se desarrolló en el Perú la filoxera literaria, o sea pasión febril por la literatura". Luego explica: "Al largo período de revoluciones y motines, consecuencia lógica de lo prematuro de nuestra Independencia, había sucedido una era de paz, orden y garantías. Fundábase planteles de educación; la Escuela de Medicina adquiría prestigio impulsada por su ilustre decano don Cayetano Heredia; y el Convictorio de San Carlos bajo la sabia dirección de don Bartolomé Herrera, reconquistaba su antiguo esplendor”. Relata también que por entonces llegó a nuestro país don Sebastián Lorente, importante figura a la que "la nueva generación seguía y escuchaba como a un apóstol ” (Palma 1971:7).

Basadre refiere la abundancia de obras y discusiones que tuvieron la virtud de llamar la atención por los fenómenos literarios, así como la publicación de antologías tanto nacionales como extranjeras y muchas formas de propaganda de sus ideas. Y precisa que a los miembros del romanticismo peruano "pese que produjeron teatro y en menor medida novela, la poesía los atrajo preferentemente". En el tomo VI de su Historia de la República, bajo el subtítulo El proceso del romanticismo peruano, Basadre vuelve al tratar el tema y lo hace glosando a José Miguel Oviedo : 
“J.M.O, joven crítico...ha planteado la tesis del fracaso del romanticismo en el Perú. Reacciona con ella contra las actitudes indulgentes o superficiales de la crítica literaria tradicional. Oviedo sostiene que no hubo aquí verdadera escuela romántica, que la llamada bohemia se disgregó después de una militancia corta...que las mejores expresiones de sus personeros no siempre pueden ser adscritas a dicha escuela, y que, en conjunto, el romanticismo en el Perú presenta un movimiento débil... Por otra parte, pone de manifiesto los malos hábitos literarios del romanticismo nacional que no creó un estilo propio, no tuvo un gesto de verdadera independencia estética y exhibió pobreza verbal e imaginativa, desorden, mal gusto, incapacidad paisajística y también alejamiento de las raíces sociales demostrado en el olvido de la obra de Melgar y en el desdén ante el legado que ella dejó" (Basadre 1965: VI 305).

El historiador peruano deslinda entre la historia cultural y la historia social. Saluda la existencia en cada nueva generación "las podas y las campañas de saneamiento en la historia cultural, distinta de la historia social" y añade: "El historiador general puede convenir en el atraso de la producción romántica peruana y en sus múltiples fallas ... A pesar de todo, inquietud, fervor, animación aparecen en los días de Salaverry, Corpancho, Luis Benjamín Cisneros, Márquez, Palma y sus contemporáneos, más que en los años anteriores" (Basadre 1965: VI 306). Anteriormente había esbozado el tema de los criterios que deben regir el enjuiciamiento a una obra o el conjunto de obras: "En el mundo de la estética la posteridad juzga de acuerdo con premisas y criterios que quienes son juzgados no pudieron imaginar".

Críticos contemporáneos coinciden en sus juicios acerca de esta etapa de la literatura en el Perú. Ricardo Silva Santisteban sostiene lo siguiente: "Por su tibieza nuestro romanticismo carece de una verdadera poética. No reconocemos en él ni nuestra vida ni nuestro paisaje ni nuestros problemas... es un pobre reflejo del ya pobre romanticismo español” (1984: 18). Mientras que Carlos García Bedoya señala que "el romanticismo peruano se caracterizó por la inautenticidad y domesticación, conservando mucho de la retórica neoclásica. El género más frecuentado fue la lírica, escribiéndose también unas pocas novelas, producción toda ella de escasa calidad" (1990:86). 


\section{El romanticismo en los estudios de Historia de la Literatura Pe- ruana}

Las visiones panorámicas de la literatura de un país son para el común de los lectores, obras inamovibles, resultado de amplio consenso, sus contenidos consagran nociones con respecto a un autor, una obra o una etapa. Sin embargo, como señala Washington Delgado "la historia es una perspectiva... no hay una historia neutra... En el caso de la literatura hay que señalar además que no solamente la aparición de nuevas obras literarias modifica cuantitativamente la historia, en cuanto la prolonga en el tiempo, sino que también nuevas obras modifican la perspectiva desde la cual contemplamos las obras anteriores" (1980:13). No obstante, para entender el romanticismo peruano puede ser útil revisar lo expresado por historiadores de la literatura nacional, con especial atención a la mención que hacen de la obra de algunos de los integrantes del grupo de los románticos que pudieran no haber sido suficientemente estudiados.

Por ejemplo, Luis Alberto Sánchez, afirma: "Podría decirse que el romanticismo peruano fue producto tanto del temperamento y ambiente locales, como de la atmósfera emotiva que predominaba en el continente, y, también, de la moda europea” (1989:III,1246). Explicará que aunque durante el gobierno de Castilla, "primer presidente que realizó la proeza de durar”, los escritores podían exponer sus puntos de vista sin ser obstaculizados por el poder político, sus opiniones no tuvieron mayor influencia ni representó un desafío a la sociedad plutocrática. Por ello remarca la superficialidad de las posturas "no tanto como protesta contra la sociedad burguesa, sino como aceptación de la influencia o hegemonía europea"(1248). Analizando los elementos que llama leyendismo y exotismo, advierte que los románticos peruanos representaron al indio en sus obras pero "no se trataba del indio problema, sino del indio espectáculo... personaje tan exótico como si, en lugar suyo, se colocase un turco de Estambul en plena jungla amazónica; un pirata de Espronceda en el lago Titicaca”(1258). Respecto al lenguaje Sánchez menciona que libres de las limitaciones extraliterarias, los autores cedieron al impulso retórico y las exageraciones expresivas, pero reconoce que esto sucedió no sólo en el país. Soledad, tristeza y desventuras aparecen en 
todas las voces. Entre ellas Sánchez distingue la desventurada y singular vida de Arnaldo Márquez "uno de los pocos poetas peruanos de aquel tiempo con derecho a increpar a la suerte" (1271).

Para Cornejo Polar "nuestros románticos repitieron temas y formas propios de los maestros franceses, españoles y -con menor asiduidadalemanes" (1980: 33). Destacará, sin embargo, que "pese a sus limitaciones,... con esta poesía se establece entre nosotros una correlación definitoria entre el quehacer poético y la expresión de la subjetividad humana”(33). Más adelante advierte la existencia de tres corrientes que es posible distinguir. La ya indicada intimista, otra de carácter patriótico "nutrida con la victoria del dos de mayo de 1866, y una tercera de raíz reflexiva "si se quiere, filosófica" (33). En esta última, ubicará la obra de Arnaldo Márquez.

Augusto Tamayo Vargas indica que "la etapa del romanticismo peruano avanza desde 1940 hacia 1900, perdurando en medio de los embates naturalistas, del fervor parnasiano y de los primeros atisbos modernistas"(1992: 467). Considera que gran parte de la producción es "exótica" aun cuando se toquen temas americanos, y esto lo explica porque "la influencia se hace viva y palpitante a través del siglo XIX, reproduciendo - tardíamente - el penacho nacionalista que surge con los ejércitos napoleónicos; el lamentar angustioso que se mantiene después de su ocaso y muerte; y el renacer de la desesperación de vivir que creara la generación alemana de Goethe. Después vendrá la burlona risa escéptica y la prédica social" (467). Pese a esto sostiene que "el periodo romántico, tan vulnerable en muchos aspectos, corresponde a la primera generación que se propuso trabajar seriamente y que comprendió que el arte, la expresión literaria, responde a un oficio. Que, por encima del gesto de la libertad, hay que entender la disciplina del estudio y el ejercicio técnico con un planteamiento científico de la literatura" (469).

\section{El grupo de los románticos}

Para Ricardo Palma, el núcleo de esta generación lo constituye "Arnaldo Márquez, Nicolás Corpancho, Adolfo García, Numa Pompilio Llona, Clemente Althaus, Luis Cisneros, Carlos Augusto Salaverry, Enrique Alvarado, José Antonio Lavalle, Mariano Amézaga, Francisco Laso, Juan 
Arguedas, Trinidad Fernández, Toribio Mancilla, Asisclo Villarán, Juan de los Heros, los hermanos Pérez (sic), Narciso Aréstegui, y dos o tres nombres más quienes hacían sus primeros versos y borroneaban su primera prosa, desde los claustros del colegio" (Palma, 1971: 8) . Más adelante alcanza información en torno a la actitud vital del grupo y sus lecturas literarias : “...arrastrados por el libérrimo romanticismo, nosotros desdeñábamos todo lo que a clasicismo tiránico apestara y nos dábamos un hartazgo de Hugo y Byron, García Tassara y Enrique Gil. Arnaldo Márquez se sabía de coro a Lamartine; Corpancho no equivocaba letra de Zorrilla, Llona se apasionaba con Leopardi” (8).

Sumamente crítico con el movimiento en general, "moda o enfermedad, tuvo en el Perú caracteres de una intoxicación”, Raúl Porras Barrenechea, refiere que "se congregaban en casa del mecenas Miguel del Carpio, colaboraban en la Revista de Lima, que dirigió José Antonio de Lavalle y se retrataron con patillas y corbatas en 1830 en el Parnaso Peruano de Cortés". Y distingue algunos nombres como "los más significativos del romanticismo peruano Carlos Augusto Salaverry, Luis Benjamín Cisneros, Ricardo Palma, Arnaldo Márquez, Manuel Adolfo García, Clemente Althaus y Juan de Arona”(1969: 49-50).

\section{Las antologías}

Como se ha mencionado, un aspecto importante en la difusión de las obras de algunos de estos escritores fueron los trabajos antológicos. Por ejemplo, El Parnaso Peruano de José Toribio Polo es para Cornejo Polar (1989:46-47), la primera de una serie de antologías "específicamente literarias”. En ella, como reza el subtítulo: Repertorio de poesías nacionales antiguas y modernas ${ }^{2}$ se presenta una nómina que va de Ruiz, Caviedes, Peralta Melgar y Olavide, hasta los románticos como Palma, Márquez o Salaverry. En este trabajo, para Cornejo Polar, se establece un primer criterio de periodización puesto que el antólogo refiere que en materiales acumulados por tres siglos se hallan dos grandes momentos. El primero lo denomina místico - erudito y el segundo es ponderado como propiamente artístico. De los más jóvenes de los seleccionados dirá el antólogo que "cantan arrastrados por las corrientes eléctricas que nos

2 Antonio Cornejo Polar, en La formación de la tradición literaria del Perú, cita que fue editada en Lima, en la Imprenta La Epoca, 1862. 
envía el viejo mundo en sus libros de Goethe, Schiller, Byron, Espronceda, Lamartine y Víctor Hugo”. Años después, en 1971, aparece otro Parnaso Peruano , publicado en Valparaíso por José Domingo Cortés. Como reseña Cornejo Polar a pie de página, esta nueva antología "incluye 44 poetas, desde Larriva, Pardo o Valdez hasta - el más joven - Márquez, nacido en 1846" (Cornejo 1989: 49).

\section{José Arnaldo Márquez, el de la vida más romántica}

José Arnaldo Márquez fue, indudablemente, el de la vida más romántica. Poeta, ensayista, autor teatral, unió a sus actividades literarias el ser un viajero impenitente, inventor de una máquina de linotipia que no pudo patentar a tiempo. Fue maestro en Chile y Argentina, traductor exitoso de Byron, Shakespeare, Longellow y Whitman, lo que demuestra su rigor intelectual y la visión universal de la literatura y el arte.

Nacido en Lima en 1830, desde las aulas del Convictorio de San Carlos, empezó su carrera a los 18 años con una obra teatral de muy escaso mérito. Posteriormente, como refiere Vargas Ugarte, ingresó en el ejército y alcanzó el grado de sargento mayor; fue secretario del presidente Echenique y cónsul del Perú en Veracruz, México y Centro América (1986: VI,1-3). Al producirse la revolución que derribara a Echenique sufre dificultades políticas, pero cuando el gobierno de Castilla decreta una amnistía, Márquez es nombrado cónsul en Nueva York. Había publicado Poesías (Lima, 1853), La Ramoniada (Valparaíso,1955), y el poema La Humanidad, dedicado a Vigil (Lima, 1856).

Siendo cónsul en Nueva York, ante las informaciones de un ataque español al Perú, intervino en la construcción de dos buques de guerra. El contrato fue objetado por el gobierno peruano y en 1864 se vio obligado a dejar la carrera y quedó abandonado en el extranjero. Igual suerte había sufrido Carlos A. Salaverry. En 1862 habían aparecido en Lima dos volúmenes de sus Notas perdidas y, entre 1857 y 1862, sus Recuerdos de un viaje a los Estados Unidos. Al producirse el ataque español, Márquez se halla en E.E.U.U. y publica El Perú y la España moderna.

Durante algunos años recorrió Francia y España y de retorno a América vivió en Puerto Príncipe, Argentina y Chile. En cada uno de estos lugares fundó liceos. Por esa etapa ideó una máquina de linotipia que 
"representaba para el poeta la fortuna y la tranquilidad, pero que sólo le da quebraderos de cabeza” (Arriola 1988: 311-312). Este hecho es relatado por Martín García Merou, escritor argentino citado en el diccionario de Milla Batres, en la ficha dedicada a Márquez: "me refirió su triste odisea de soñador vagabundo. Había hecho o creído hacer un invento en el que cifraba sus más gratas esperanzas. Era una máquina para componer e imprimir con un número reducidísimo de tipos de una manera mecánica”. Luego relata las peripecias que vivió para poner en práctica su idea. Finalmente, logra patentar su invento en Nueva York en 1873 y estuvo a punto de fabricarlo en el Perú después de múltiples gestiones que lograron que el Congreso Peruano se pronunciara en ese sentido el 18 de enero de 1879. Lamentablemente al estallar la Guerra del Pacífico su sueño quedó truncado.

La producción literaria de Arnaldo Márquez abarca diversos géneros. Destaca, además, su tarea de traductor. Tamayo Vargas menciona que "en los años 1883 y 1884 salieron a luz sus traducciones de Shakespeare... aparecieron en Barcelona el drama Julio César y la comedias Las alegres comadres de Windsor, Como gustéis y Comedia de las equivocaciones, para ofrecer después El sueño de una noche de verano, Medida por medida, Coriolano y Cuento de invierno. Se trataba de un encargo de la Real Academia Española "(1992: 499). Citando a Estuardo Núñez, Tamayo Vargas refiere las traducciones de Márquez a Byron, Longfellow y Whitman. Terminada la guerra, en 1889, publicó el ensayo La orgía financiera del Perú (El guano y el salitre). Su obra completa, bajo el título Prosa y verso, apareció en dos volúmenes en 1901 y 1902. Sus últimos días transcurrieron en el Hotel Central, de la calle Palacio, en Lima. Murió en medio de la pobreza el 6 de diciembre de 1993. Hacía sólo tres meses que había podido regresar al Perú, gracias a la ayuda de Eduardo López de Romaña.

\section{Márquez y el romanticismo en la vertiente filosófica y social}

La obra de Arnaldo Márquez, diversa en géneros y temas, presenta, sin embargo, una línea que le da continuidad y coherencia. Se trata de la actitud de reflexión filosófica que conjuga solidaridad humana y apelación a altos ideales. Estos rasgos de su producción llevan a Washington Delgado a recomendar: "Hay algunos poetas románticos que 
en un trabajo más exhaustivo de la historia literaria merecerían consideración, como Arnaldo Márquez... el único de nuestros poetas de la época que desarrolló la veta humanitarista y vagamente socializante" (1980:65-66).

Tamayo Vargas y Cornejo Polar coinciden en ubicar su poesía en la corriente filosófica del romanticismo peruano al lado de Luis Benjamín Cisneros. Cornejo Polar enfatiza: "sobre todo el poco estudiado José Arnaldo Márquez, a quien Riva Aguero trató con injusta y sintomática displicencia”(1980:36). Cornejo añade que en su poética se da un generoso sistema de valores éticos y examen de la realidad circundante, destacando que "aquellos (los valores) se basan en principios religiosos, pero enfatizan su realización material tanto en referencia a la armonía de la naturaleza cuanto en relación a la justicia que debe reinar en la vida social" (1980:36). Para Ricardo Silva Santisteban - crítico severo del romanticismo - "Márquez posee un buen verso y hubiera estado llamado a ser nuestro poeta romántico por antonomasia” (1984:447). Y aunque discrepa del "tono moralista" de su poesía, señala luego que "en algún momento se supera a sí mismo y pulsa cuerdas que aún nos emocionan” (1984: 447).

En el poema "Noche de luna" (Santiago de Chile, 1849) título expresivo de la corriente romántica, queda patentizada la serena contemplación del paisaje en armonía plena con el yo poético. Naturaleza que sirve de marco al pensamiento y el sueño: "Luz tenue y melancólica/ de lánguida dulzura /colora tibiamente /la blanca vestidura / que tiende sobre el valle / levísimo vapor /.../ Qué bellas esas aguas / donde luz riela / y en cuyas quietas márgenes / dormita la ciudad ! / sobre su sueño el ángel / de los misterios vela / mientras el tiempo rápido / sobre su frente vuela / como una tibia ráfaga / de suave claridad." // Oh sí ! Todo es hermoso / La noche, el firmamento / los astros y las nubes / convidan al placer. / En medio de memorias / dormita el pensamiento / y el alma a sus ensueños / entregase un momento / cual hoja que en los aires / fluctúa sin caer !".

En sus "Leyendas peruanas" encontramos la señalada característica filosófica que trasciende el marco individual e ilumina de valores expansivos todo fenómeno : "No sabe lo que es la vida / quien nunca amó, ni comprende / cómo ella el instinto enciende / de bien en el corazón / y 
busca en torno otros seres / que lo amen y lo bendigan / prodigándoles placeres / en generosa expansión".

Tópicos recurrentes en el arte romántico, la noción del artista como un ser predestinado a la creación, y la idea de la "realidad no poética" se encuentran en estos fragmentos del poema "Mi poesía": "No hay duda es mi destino / que yo también creara esa armonía / vestigio de un remoto paraíso / que llama el universo poesía.// Fuera de ella mi espíritu indeciso / se agita en una atmósfera vacía / donde no encuentra ni una luz siquiera / que alumbre y guíe su fugaz carrera”. Interesante además la idea del arte capaz de comunicar diversas esferas. Dios, amor y belleza captados y transmitidos por la palabra poética con una evidente revaloración de la naturaleza en tanto gratuidad y espontaneidad frente a las normas neoclásicas, expresadas en otras estrofas del mismo poema. "Todo en el mundo para mí es un canto / todo en la vida es para mí un acento / que hablan de un ser incomprensible y santo / que no puedo mirar, pero que siento / el orbe lo saluda, y entre tanto / le habla la vida con su gran lamento; / y entrambas voces que a la par se elevan / un mismo nombre al infinito llevan”.

Finalmente, en su poema titulado "La vida", fechado en diciembre de 1875 y publicado en la segunda edición de sus Notas perdidas, en 1878, destacan las ideas de brevedad de la existencia, el dolor como forma de conocimiento y la noción de trascendencia como única salida al inevitable deterioro de la vida terrena. Reproducimos aquí la versión seleccionada en la antología de Ricardo Silva Santisteban: "La vida es como un árbol del camino / cuando soplan las brisas del otoño/ .../ Los deleites, los sueños, la alegría / son las hojas pintadas de la copa / que hace caer al declinar el día / la brisa melancólica y fugaz./ .../ Así también al desnudar los años / de ilusiones y goces nuestra vista / descubren ante el alma entristecida/ pedazos de su inmenso porvenir ".

Con esta breve muestra de la obra poética de Arnaldo Márquez, queremos poner de manifiesto algunas particularidades con las que este poeta peruano, insuficientemente estudiado, se inscribe dentro de la tendencia romántica. Poética que reflexiona sobre el destino del hombre y el destino del universo. En esa dirección se halla su preocupación por la justicia y el permanente afán por entender mejor los hechos de su momento histórico, que motivaron también sus ensayos. 


\section{Referencias bibliográficas}

ANDERSON IMBERT, Enrique (1995). "La colonia. Cien años de República” En: Historia de la Literatura Hispanoamericana. México: Fondo de Cultura Económica.

ARRIOLA, Maurilio (1988). “José Arnaldo Márquez” En: Diccionario Literario del Perú. Barcelona: Comercial de Artes Gráficas, pp. 311-312.

BASADRE, Jorge (1965). Historia de la República del Perú. T. IV y VI. Lima: Editorial Universitaria UNMSM.

CORNEJO POLAR, Antonio (1980). "Literatura en el Perú Republicano". En: Historia del Perú. Perú Republicano y Procesos e Instituciones. T. VIII, Lima: Editorial Juan Mejía Baca.

CORNEJO POLAR, Antonio (1989). La formación de la tradición literaria en el Perú. Lima: CEP.

DELGADO, Washington (1980). Historia de la Literatura Republicana. Lima: Rikchay Perú.

ESCOBAR, Alberto (Prólogo, selección y notas) (1958). Salaverry, poesía. Lima: UNMSM.

GARCÍA BEDOYA, Carlos (1990). Para una periodización de la Literatura Peruana. Lima: Latinoamericana editores.

MOTA, Francisco (1977). Poetas españoles de la generación del 27. La Habana: Biblioteca Básica de Literatura española.

PALMA,Ricardo (1971). La bohemia de mi tiempo. Lima: Librería y distribuidora Bendezú.

PORRAS BARRENECHEA, Raúl (1969). El sentido tradicional en la literatura peruana. Lima: Instituto Raúl Porras Barrenechea de la UNMSM.

SALAZAR BONDY, Sebastián (Selección y notas) (1956). Mil años de poesía peruana. Lima: Populibros peruanos.

SÁNCHEZ, Luis Alberto (1989). La Literatura Peruana. Sexta edición, T. III, Lima: EMISA.

SILVA SANTISTEBAN, Ricardo (1984). Poesía peruana. De la conquista al modernismo. T. II, Lima: Edubanco.

TAMAYO VARGAS, Augusto (1992). Literatura Peruana, T. II, Lima: PEISA. 
VARGAS UGARTE, Rubén (1986). "Arnaldo Márquez". En Diccionario Histórico Biográfico del Perú, T. VI, Lima: Milla Batres.

ZANUTELLI ROSAS, Manuel (1994). “José Arnaldo Márquez”. En Enciclopedia Biográfica e Histórica del Perú. Siglos XIX-XX. T. VI. Lima: Editorial Milla Batres. 